\title{
PREDICTION OF NONLINEAR SYSTEM IN OPTICS USING GENETIC PROGRAMMING
}

\section{7

\author{
AMR RADI \\ Physics Department \\ Ain Shams University, Cairo, Egypt \\ Amr1165@yahoo.com
}

Received 26 May 2006

Accepted 31 May 2006

\begin{abstract}
It is difficult to predict the dynamics of systems which are nonlinear and whose characteristic is unknown. In order to build a model of the system from input and output data without any knowledge about the system, we try automatically to build prediction model by Genetic Programming (GP).

GP has been used to discover the function that describes nonlinear system to study the effect of wavelength and temperature on the refractive index of the fiber core. The predicted distribution from the GP based model is compared with the experimental data. The discovered function of the GP model has proved to be an excellent match to the experimental data.
\end{abstract}

Keywords: Genetic programming; refractive index; temperature; wavelength; nonlinear.

\section{Introduction}

Most systems in the real world are essentially nonlinear and time-dependent. ${ }^{1}$ We are sometimes required to deal with systems whose input and output are known but the mechanism is unknown. Artificial Neural Networks (ANN) and polynomials are two methods for global modeling. ${ }^{9}$ But both cannot give simple and elegant model representations. Essentially speaking, they are more appropriate to be regression and approaching tools than modeling tools. They are less powerful in revealing the system dynamic laws and are difficult to integrate with the pre-discovered knowledge on nonlinear systems. ${ }^{7}$

This paper presents a genetic programming (GP) model which has been one of research interest in modeling high energy physics and in automated re-invention of six patented optical lens systems. ${ }^{8,11}$ As well, $\mathrm{GP}^{7}$ have succeeded in the field of automatic define function. ${ }^{2}$ Oakley used GP to evolve equations to fit the chaotic time series produced by Mackey-Glass equations. ${ }^{3}$ Iba, Kurita, de Garis and Sato used GP for system identification problems. ${ }^{4}$

As in Ref. 10, researchers try to obtain the refractive index of the core of the fiber as a function of wavelengths $\lambda$ at different temperature $T$ but they cannot 
Table 1. The fitting parameters of the Sellmeier equation for the core at different room temperatures $\left(E=100 \mu \mathrm{m}^{2}\right)$.

\begin{tabular}{ccccc}
\hline Temp. ${ }^{\circ} \mathrm{C}$ & $A$ & $B$ & $C$ & $D$ \\
\hline 20 & 1.331522 & 0.827246 & 0.011065 & 0.999995 \\
25 & 1.329672 & 0.829166 & 0.011049 & 0.985995 \\
29 & 1.328192 & 0.830559 & 0.011037 & 0.974795 \\
34 & 1.326342 & 0.832615 & 0.011021 & 0.960795 \\
40 & 1.324122 & 0.834929 & 0.011003 & 0.943995 \\
\hline
\end{tabular}

1

3

5

achieve a function which depends on wavelengths and temperatures. Making use of the capability of GP, the present work uses GP to model the refractive index as a function of wavelength and temperature. GP is fed with wavelengths and temperature while the output is the refractive index.

The following sections provide a brief introduction to the two-pole Sellmeier dispersion formula, GP, describe the selected GP structure and discuss the results.

\section{Two-Pole Sellmeier Dispersion Formula}

An accurate dispersion formula (find the refractive index $n$ ) that can be applied over a wide range of wavelengths $\lambda$ is the two-pole Sellmeier dispersion formula in the form ${ }^{9}$

$$
n^{2}(\lambda)=A+\frac{B}{1-\left(C / \lambda^{2}\right)}+\frac{D}{1-\left(E / \lambda^{2}\right)}
$$

$\lambda$ is the wavelength measured in micrometers. Here $A, B, C, D$, and $E$ are called the dispersion parameters of the fiber core materials ( $E$ does not depend on wavelength or temperature).

In Ref. 10, they applied the two-pole Sellmeier dispersion formula to the core of the fiber at different room temperatures as in Table 1. My proposal is to find a function which represents the refractive index $n(\lambda, T)$ depending on wavelengths and temperature. The function should prove to be a better match for experimental data (i.e., less than the experimental error of 0.001).

\section{Modeling by GP}

\subsection{Model building}

It is easy to build a prediction model using GP because it has many advantages: to handle mathematical modelling is easy, special knowledge is not needed to search a solution and it can cope with the change of various conditions.

GP handles tree-structured chromosome that represents a mathematical model of the system, see Fig. 1. Nodes involved in the tree-structured chromosome belong to two categories: one involves input data (leaves), the other involves operators $(+,-, *, \ln , \ln 2, \cos , \sin )$ (inner nodes of the tree). 


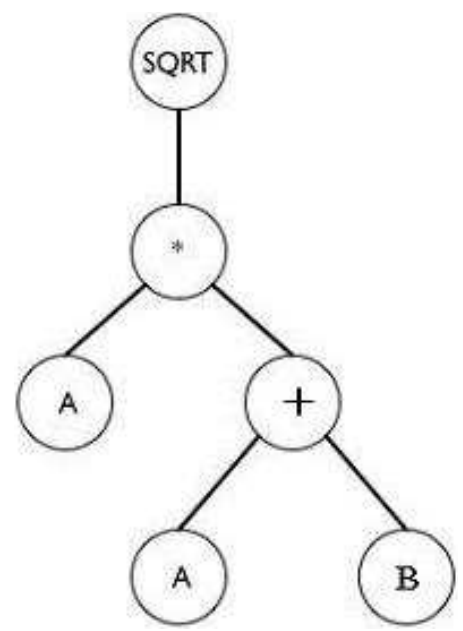

Fig. 1. Experimental and trained refractive index dispersion formula of the core of the fiber.

\subsection{Procedure of GP}

$\mathrm{GP}^{5-7}$ evolves from a population of computer programs, which are possible solutions to a given optimization problem, using the Darwinian principle of survival of the fittest. It uses biologically inspired operations like reproduction, crossover and mutation. Each program or individual of the population is generally represented as a tree composed of functions and data/terminals appropriate to the problem domain. The input set of functions and terminals have to satisfy the closure and sufficiency properties. The sufficiency closure property requires that the set of functions and the set of terminals are able to express solution of problems. The function set may include standard arithmetic operators, logical operators, mathematical functions and specific functions. The terminal set usually consists of feature variables and constants. Each individual in the population is assigned a fitness value, which quantifies how well it performs in the problem environment. The fitness value is computed by a problem dependent fitness function.

A typical implementation of GP involves the following steps.

(1) GP begins with a randomly generated population of solutions of size.

(2) A fitness value is assigned to each solution of the populations.

(3) A genetic operator is selected probabilistically as follows:

Case i If it is the reproduction operator, then an individual is selected (we use fitness proportion-based selection) from the current population and it is copied into the new population. Reproduction replicates the principle of natural selection and survival of the fittest.

Case ii If it is the crossover operator, then two individuals are selected. We use tournament selection where a number of individuals is taken randomly from the current population, and out of these, the best two individuals (in terms 
4 A. Radi

of fitness value) are chosen for the crossover operation. Then, we randomly select a sub tree from each of the selected individuals and interchange these two subtrees. The two offsprings are included in the new population. Crossover plays an essential role in the evolutionary process.

Case iii if the selected operator is mutation, then a solution is (randomly) selected. Now, a subtree of the selected individual is randomly selected and replaced by a new randomly generated subtree. This mutated solution is allowed to survive in the new population. Mutation maintains diversity.

(4) Continue step 3, until the new population gets solutions. This completes one generation.

(5) If GP has not yet converged, steps 2-4 are repeated till a desired solution (maybe 100\% correct solution) is achieved. Otherwise, terminate the GP operation after a predefined number of generations.

\subsection{The proposed genetic programming}

The author's approach is to use the experimental data ${ }^{10}$ (wavelengths $\lambda$ and temperature $T$ ) to produce the refractive index for each case. The wavelengths $\lambda$ and temperature $T$ are used as input variables to find the suitable function that can describe the experimental data.

The fitness function is calculated as a negative value of the total absolute performance error of the discovered function on the experimental data set, i.e., a lower error must correspond to a higher fitness. The total performance error can be defined for all the experimental data $(i=1, \ldots, n)$ set as:

$$
E=\sum_{j=1}^{n}\left|X_{j}-Y_{j}\right|^{2}
$$

where $X_{j}$ represents the experimental data for element $j$ and $Y_{j}$ represents the calculated data for element $j$. The running process stops when the error $E$ is reduced to an acceptable level (0.00001).

\section{Results}

Our representation GP was run for 500 generations with a maximum population size of 900 . The operators (and selection probability) were: crossover with probability 0.8 and mutation with probability 0.01 . The function set is $\left(+,-, *, /, \ln , \log _{2}, \sin , \cos \right)$, and the terminal set is (random constancy from 0 to $10, \lambda, T$ ). the "full" initialisation method was used with an initial maximum depth of 27 , and tournament selection with a tournament size of 8 .

Inputs training data are the wavelengths $\lambda=300-700 \mathrm{~nm}$, and temperature $T=20,25,29,34$ in ${ }^{\circ} \mathrm{C}$. Each refractive index $n$ corresponds to certain wavelengths $\lambda$ at different temperature $T$. The discovered function (in the Appendix) has been trained to associate the input patterns to the target output patterns for 

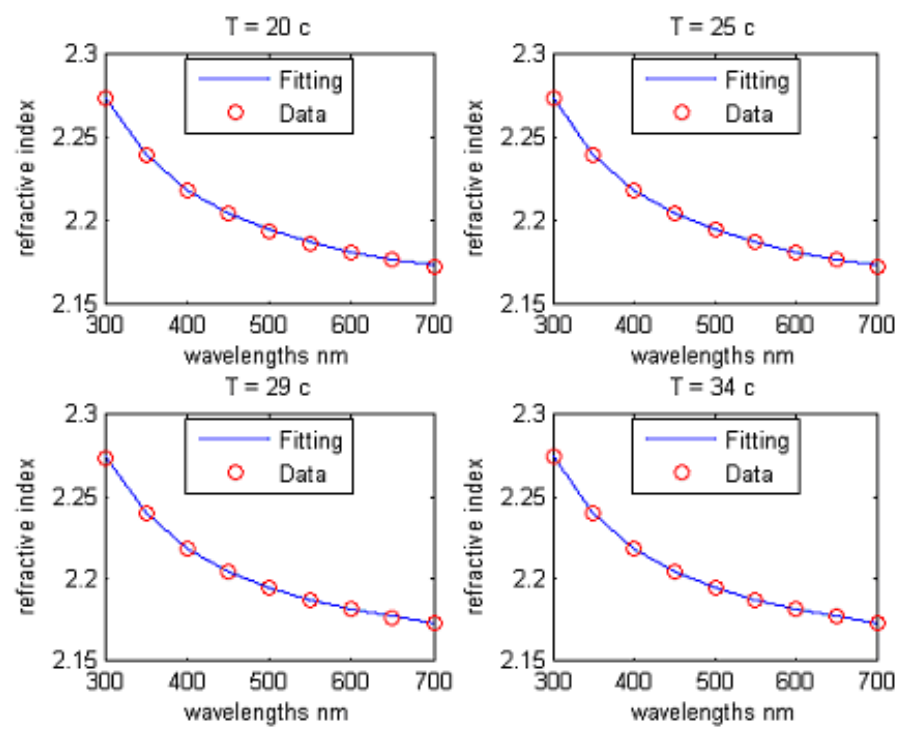

Fig. 2. Experimental and trained refractive index dispersion formula of the core of the fiber.

FIGURE 3

Fig. 3. Experimental and predicted refractive index dispersion formula of the core of the fiber.

1 the above wavelengths. Figure 2 illustrates the experimental and trained refractive index. After running the GP, the discovered function has been used to predict 3 the refractive index, corresponding to $T=40^{\circ} \mathrm{C}$ with the same $\lambda=300-700 \mathrm{~nm}$. Figure 3 illustrates the experimental data and predicts the refractive index.

\section{Conclusions}

Genetic programming has been run to model the dispersion formula depending on

7 the temperature. GP discovered a function which represents the dispersion formula at different temperatures. The discovered function shows an excellent match to 
the experimental data. Moreover, the discovered function is capable of predicting experimental data for dispersion formula that are not used in the training session. Finally, we conclude that GP have become one of the important research areas in the field of optics.

5

7

9

\section{Appendix}

$*\left(+\left(\ln \left(\sin \left(\ln \left(-\left(/\left(\log _{2}(+(/(/(\ln (\ln (\lambda)), 10), \ln (0.72349)), \lambda))\right.\right.\right.\right.\right.\right.\right.$, $\log _{2}\left(/\left(\log _{2}(\lambda),-\left(/\left(\log _{2}\left(+\left(/\left(/\left(\ln \left(\ln \left(-\left(\log _{2}(\lambda), 10\right)\right)\right), 10\right), \ln (0.72349)\right)\right.\right.\right.\right.\right.\right.$,

$\left.\left.\left.\left.\left.\left.\left.\left.\left.\lambda)), \log _{2}\left(/\left(\log _{2}(\lambda), 10\right)\right)\right), 10\right)\right)\right)\right), 10\right)\right)\right)\right),-\left(\cos \left(\cos \left(\sin \left(/\left(\log _{2}(+\right.\right.\right.\right.\right.$

$\left(/ /(\ln (\ln (\lambda)), 10), \ln \left(\sin \left(\ln \left(-\left(/\left(\log _{2}(+(/(/(\ln (\ln (\lambda)), 10)\right.\right.\right.\right.\right.\right.$,

$\ln (0.72349)), \lambda)), \log _{2}\left(/\left(\log _{2}(\lambda),-\left(/\left(\log _{2}\left(+\left(/ / /\left(\ln \left(\ln \left(-\left(\log _{2}(\lambda)\right.\right.\right.\right.\right.\right.\right.\right.\right.\right.$,

10)) $\left.\left.\left.\left.\left.\left.\left.\left.\left.\left.\left.\left.), 10), \ln (0.72349)), \lambda)), \log _{2}\left(\left(\log _{2}(\lambda), 10\right)\right)\right), 10\right)\right)\right)\right), 10\right)\right)\right)\right)\right), \lambda\right)\right)$,

$\left.\left.\left.10)))), \ln \left(*\left(\log _{2}(0.85892), \sin (0.72349)\right)\right)\right)\right), /(T, *(*(\lambda, 0.67269), T))\right)$.

\section{References}

1. K. J. Astrom and P. Eykhoff, System identification, a survey, Automatica 7 (1971).

2. X. C. Jian and J. L. Zheng, A chaotic global modeling method based on orthogonal polynomials, Acta Electronica Sinica 30, 76 (2002).

3. I. Yoshihara and S. Sato, Nonlinear model building method with GA and GMDH, IPSJ, AI-105, 1-6 (1996) (in Japanese); Advances in Genetic Programming, ed. K. E. Kinnear, Jr. (MIT Press, 1994).

4. H. Iba, H. deGaris and T. Sato, Recombination guidance for numerical genetic programming, in Proc. of 2nd International Conference on Evolutionary Computation (IEEE Press, 1995).

5. J. R. Koza, Genetic Programming: On the Programming of Computers by Means of Natural Selection (MIT Press, Cambridge, MA, 1992).

6. W. Banzhaf, P. Nordin, R. E. Keller and F. D. Francone, Genetic Programming: An Introduction: On the Automatic Evolution of Computer Programs and Its Applications (dpunkt.verlag and Morgan Kaufmann, 1998).

7. J. R. Koza, M. A. Keane, M. J. Streeter, W. Mydlowec, J. Yu and G. Lanza, Genetic Programming IV: Routine Human-Competitive Machine Intelligence (Kluwer Academic Publishers, 2003).

8. J. M. Link, (FOCUS), Application of Genetic Programming to High Energy Physics Event Selection, Nucl. Instrum. Meth. A 551, 504 (2005), hep-ex/0503007.

9. G. C. Bhar, Applied Optics 15, 305 (1976).

10. M. Medhat, S. El-zaiat, A. Radi and M. Omar, Application of fringes of equal chromatic on optical parameters of a GRIN optical fiber, J. Optics A: Pure Applied Optics. 4, 174 (2002).

11. J. R. Koza, S. H. Al-Sakra and W. J. Lee, Automated re-invention of six patented optica lens systems using genetic programming, Genetic and Evolutionary Computation Conference (GECCO) '05 (Washington, DC, 2005). 